Mots. Les langages du politique

Le sigle comme modalité de dissociation énonciative. Le cas des alternances d'usage PMA / Pays les moins avancés

Françoise Dufour

\title{
OpenEdition
}

Journals

Édition électronique

URL : https://journals.openedition.org/mots/20112

DOI : $10.4000 /$ mots. 20112

ISSN : 1960-6001

Éditeur

ENS Éditions

Édition imprimée

Date de publication : 1 mars 2011

Pagination : 75-87

ISBN : 978-2-84788-307-7

ISSN : 0243-6450

Référence électronique

Françoise Dufour, « Le sigle comme modalité de dissociation énonciative. Le cas des alternances d'usage PMA / Pays les moins avancés ", Mots. Les langages du politique [En ligne], 95 | 2011, mis en ligne le 01 mars 2013, consulté le 22 avril 2022. URL : http://journals.openedition.org/mots/20112 DOI : https://doi.org/10.4000/mots.20112 


\section{Le sigle comme modalité de dissociation énonciative. Le cas des alternances d'usage PMA / Pays les moins avancés}

La siglaison est généralement définie comme un processus d'abréviation qui procède d'une nécessité d'économie linguistique (graphique et orale). Parmi les exemples illustratifs de sigles couramment cités dans la littérature, on trouve par exemple HLM (habitation à loyer modéré), PMU (pari mutuel urbain), PTT (poste télégraphe téléphone), BEPC (brevet d’études du premier cycle) ou encore RMI (revenu minimum d'insertion). Ces sigles familiers sont solidement rattachés à des réalités sociales et à des expériences concrètes stockées dans la mémoire culturelle et autobiographique, en d'autres termes à un « référent expérientiel » (Launay, 1986, p.18). L'acte de référenciation se réalise aisément en accompagnement du signifiant des sigles. En revanche, l'énoncé des syntagmes correspondant aux sigles ne se fait pas sans difficulté. Le déploiement à partir de sigles bien connus demande un effort aux sujets sollicités, fait appel à l'improvisation et il en résulte souvent une certaine imprécision. Louis-Jean Calvet en a fait l'expérience en administrant des enquêtes de reconnaissance et de traduction de trente sigles courants à des élèves de lycée de classes d'âge différentes. Les résultats de ses enquêtes ont montré que «l'expérience concrète, l'usage quotidien du sigle sont séparés de la connaissance de sa source. [...] Le sigle semble donc avoir dans l'oralité une existence autonome, renvoyer à un signifié sans que la médiation de sa source ne soit nécessaire » (Calvet, 1980, p. 78).

C'est le résultat d'une fonction du sigle que Jacqueline Percebois nomme «la fonction dénominative lapidaire véhiculaire (FDLV) » : «La FDLV va mettre des concepts obscurs à la portée de tous; les usagers de ces sigles grand public n'ayant, le plus souvent, plus aucun contact avec les formations lexicales composées exprimant les concepts. » (Percebois, 2001, p. 631)

La difficulté d'identification du sigle à sa forme développée d'origine offre la potentialité à la forme siglée d'être le support d'une identité signifiante indépendante de sa forme source. 
J'analyserai en contexte les manifestations de ce phénomène d'indépendance de la forme siglée ainsi que les alternances d'usage qu'elle permet, avec le cas de la siglaison PMA du syntagme pays les moins avancés. Les deux formes appartiennent au discours ${ }^{1}$ politico-économique du développement : d'une part, le discours des institutions à l'origine du concept - notamment l'Organisation des nations unies (ONU) et son organe spécialisé dans le domaine du commerce et du développement, la CNUCED², ainsi que les autres institutions qui gèrent des budgets d'aide au développement et/ou octroient une assistance technique -, et d'autre part, le discours des hommes politiques qui usent de cette terminologie. Ce sont des énoncés produits par ces deux groupes de locuteurs qui constituent le corpus d'analyse de quelques cas d'alternances d'usage des deux formes - le sigle et sa forme développée - en fonction du cotexte et des contextes d'énonciation.

\section{Les usages institutionnels du sigle PMA}

\section{Le sigle en usage «décroché»: un marqueur d'identification}

La désignation d'une catégorie de pays parmi les plus pauvres de la planète par la dénomination3 "pays les moins avancés» suivie de la siglaison PMA est aujourd'hui courante dans le discours du développement. La siglaison n'a cependant pas été introduite dès la création de la catégorie des «pays les moins avancés».

Il semble que le concept d'une « sous-catégorie particulière des pays les plus démunis» (Lacharrière, 1971, p. 469) revienne au premier secrétaire exécutif de la CNUCED, l'économiste argentin Raul Prebish, à l'issue de la première conférence en 1964. La toute première mention officielle de cette potentielle catégorie de pays apparaît dans la résolution 24 (II) de la CNUCED du 26 mars 1968 :

(1) Réaffirmant son appui à la résolution 24 (II) de la Conférence des Nations unies sur le commerce et le développement en date du 26 mars 1968, par laquelle la Conférence a invité les organismes internationaux chargés des mesures particulières destinées à favoriser les pays en voie de développement d'une manière générale à concevoir et à préciser sous quelle forme ces mesures spéciales pourraient être prises en faveur des pays les moins avancés et à identifier ces pays [...].4

1. Le terme discours, employé au singulier (du discours), est ici à considérer comme « un mode de dire qui se constitue en pratique à la fois langagière et sociale» (Dufour, 2010, p. 24).

2. La Conférence des Nations unies sur le commerce et le développement (CNUCED) fondée par la résolution 1995 (XIX) du 30 décembre 1964.

3. À la suite de Paul Siblot, je distingue la nomination comme processus de la dénomination comme résultat (Siblot, 2001, p. 205-207).

4. Actes de la Conférence des Nations unies sur le commerce et le développement, $2^{\text {e }}$ session, vol. I : Rapport et annexes (publication des Nations unies, 1970, p. 58). 
La résolution 2724 (XXV) du 15 décembre 1970 officialise la requête des éléments identifiants nécessaires à la construction catégorielle :

(2) Identification des pays en voie de développement les moins avancés. 5

Enfin, en 1971, la première liste et les critères d'appartenance sont approuvés par l'ONU dans la résolution 2768 (XXVI) 6 .

La forme longue «les moins avancés des pays en voie de développement » est maintenue au fil des nombreuses résolutions qui ont adopté des mesures spéciales attribuées à cette catégorie de pays. À la fin de la décennie soixantedix, la dénomination subit une troncation et c'est la forme plus resserrée de pays les moins avancés qui est adoptée, assortie dans le même temps du sigle PMA:

(3) Depuis 1977, l'Organisation des Nations unies a dénommé «pays les moins avancés» (PMA) une catégorie de pays dont le processus de développement est entravé par un handicap structurel et qui nécessitent, dans leurs efforts de développement, un soutien très important de la communauté internationale. 7

L'abréviation en pays les moins avancés, qui donne lieu à siglaison, désolidarise le sous-groupe de sa catégorie d'ordre supérieur (les pays en voie de développement). Alors que la forme initiale «les moins avancés des pays en voie de développement» déterminait la place des pays «les moins avancés» au sein de la catégorie super-ordonnée des pays « en voie de développement», la double opération d'abréviation et de siglaison a une valeur d'acte de constitution d'une catégorie de pays à part entière.

Dans l'énoncé de la forme abrégée pays les moins avancés, l'absence de spécification de la norme à partir de laquelle est déterminée la relativité du superlatif d'infériorité les moins construit implicitement la représentation d'un pôle d'infériorité au regard de l'ensemble des pays de l'humanité en marche vers un état de développement représenté par le «type » occidental des pays «développés» ou «avancés».

Dans l'extrait (3) qui constitue l'incipit du corps du document de la CNUCED8, l'énonciateur institutionnel décline l'identité dénominative de la catégorie : «"pays les moins avancés” (PMA) » en appui sur l'acte de baptême daté (1977) et signé par l'organe de tutelle : l'Organisation des Nations unies. Est ainsi énoncée la forme officielle complète de la dénomination correspondant à la catégorie nouvellement constituée. La dénomination est composée de deux formes consécutives : un syntagme et un sigle postposé en incidente parenthétique.

5. Résolution 2724 (XXV) de l'Assemblée générale du 15 décembre 1970 [http://www.un.org/ french/documents/view_doc.asp?symbol=A/RES/2724\%28XXV\%29\&Lang=F]. Sauf indication contraire, la dernière consultation des sites mentionnés dans cet article date du 7 décembre 2010.

6. Résolution 2768 (XXVI) de l'Assemblée générale du 18 novembre 1971 [http://www.un.org/ french/documents/view_doc.asp?symbol=A/RES/2768\%28XXVI\%29\&Lang=F].

7. CNUCED, Conseil du commerce et du développement, Rapport de la $49^{\mathrm{e}}$ session, Genève, 7-18 octobre 2002, p. 3 [http://www.unctad.org/fr/docs/tb49d7.fr.pdf]. Désormais: CNUCED, 2002.

8. Après la page de titre et de résumé (p.1) et la table des matières (p. 2). 
Pour Sabine Pétillon-Boucheron, la mise en enclos que réalisent les parenthèses constitue une «opération de décrochement» : «La "mise entre" parenthèses [...] dans un ailleurs à la fois discursif et phrastique d'un segment particulier [...] nous apparaît comme le résultat d'un processus de hiérarchisation du discours dont nous ne saisissons bien évidemment que l'étape ultime, en quelque sorte la trace. »(Pétillon-Boucheron, 2003, p.127)

Cette opération apporte deux instructions de lecture : celle du «j'ajoute par ailleurs» et celle de «je le place sur un autre plan» (ibid.). L'ajout de la siglaison parenthétique fournit donc cet «autre plan» dont la détermination dépend des paramètres d'énonciation, notamment de la qualité de l'énonciateur. Dans le discours de l'énonciateur institutionnel, la mise à disposition du sigle dans l'enclos des parenthèses, à la suite de la forme syntagmatique, remplit la fonction que Jacqueline Percebois nomme «lapidaire», en référence à l'origine latine des sigles donnée par Littré comme «signes abréviatifs sur les monuments, les médailles et dans les manuscrits anciens» (Percebois, 2001, p.629-630). La mention du sigle dans le décroché des parenthèses est assimilable à un codage qui serait le garant de la certification par les autorités en état de le faire (l'ONU). Comme l'estampille9 apposée sur un objet, cette mention s'apparente à une opération d'identification garante d'une validation institutionnelle préalable de la catégorie dénommée "pays les moins avancés».

Comme dans le texte de l'ONU (3), la «communication » du Parlement européen parue au Journal officiel fait usage de la forme siglée en décroché :

(4) L'UE est par ailleurs le plus important partenaire économique et commercial des pays en développement; offrant des avantages commerciaux spécifiques à ces pays, tout particulièrement ceux qui sont les moins avancés (PMA). [...] En vue de réaliser les $\mathrm{OMD}$, la priorité sera donnée aux pays les moins avancés et aux autres pays à faible revenu (PFR) comme en témoigne la part importante de l'aide de l'UE accordée à ces pays. ${ }^{10}$

On remarque que le sigle énoncé en page 1 n'est pas repris dans la suite du texte. La seconde mention des pays de la catégorie en page 5 ne se réalise pas par la médiation du sigle. En revanche, dans le même énoncé, la mention d'une catégorie non encore évoquée dans la communication, celle des « autres pays à faible revenu », est immédiatement suivie de son code institutionnel : «(PFR)». Cette pratique qui consiste à ne mentionner le sigle en décroché qu'à la pre-

9. "Marque originale ou officielle, cachet apposé à un document écrit, à une marchandise, à un meuble, pour garantir son authenticité ou son origine, pour attester le paiement de droits fiscaux. " Trésor de la langue française informatisé (TLFi)

10. "Le consensus européen pour le développement ", Journal officiel de l'Union européenne, 2006/C 46/01, p. 1 et 5 (Déclaration conjointe du Conseil et des représentants des gouvernements des États membres réunis au sein du Conseil, du Parlement européen et de la Commission sur la politique de développement de l'Union européenne). Je souligne, dans cette citation comme dans celles qui suivent. 
mière occurrence de la catégorie et à ne pas le reprendre dans la linéarité discursive valide que le sigle ne remplit pas ici une fonction véhiculaire mais qu'il propose un autre plan.

Le décrochement du sigle peut s'analyser comme une forme de discours rapporté, celui d'un énonciateur placé comme extérieur au dire et reconnu comme l'énonciateur principal (E), auteur de la catégorisation, l'UE n'étant qu'un énonciateur second. On peut bien sûr supputer cet énonciateur comme étant l'ONU en tant qu'organisation regroupant la très grande majorité des États et par conséquent représentative de ce qu'on appelle la «communauté internationale». Mais à travers elle, c'est la sphère des institutions concurrentes et partenaires dans le système de l'aide au développement qui est reconnue comme «the principal» (Goffman, 1981, p.144), c'est-à-dire comme l'énonciateur responsable de la conformité de l'usage du sigle. Le Parlement européen fait partie de cet « univers de discours » comme «système universel de significations auquel un discours (ou un énoncé) appartient, système qui en détermine la validité et le sens» (Coseriu, 2001, p. 62), mais il ne porte pas la responsabilité pleine et entière de la dénomination qui se trouve distribuée entre les différentes institutions pourvoyeuses d'aides aux «pays du Sud».

Le sigle se présente comme une forme figée à l'usage contraint, alors que la forme syntagmatique permet plus de souplesse car elle est «déformable». En (4), la forme siglée PMA ne correspond pas à la forme antécédente : «ceux qui sont les moins avancés». La forme complète du syntagme a été défigée, pays est remplacé par le pronom ceux, anaphore de la forme antécédente pays en développement. Le retour à la catégorie des «pays en développement » réactualise l'appartenance originelle à la catégorie super-ordonnée et avec elle, le procès de création onusien.

La mention du code établi par l'ONU et validé par la communauté discursive des institutions internationales spécialisées fonctionne comme un marqueur d'identification, qui active le plan énonciatif de l'acte de signature institutionnelle.

\section{Le sigle réfère au code institutionnel, la forme syntagmatique aux actants}

Le sigle PMA sort des parenthèses pour occuper la place de la forme développée plus longue et donc plus lourde. Pourtant, les usages discursifs montrent que le choix prioritaire d'emploi du sigle ne répond pas qu'à cet impératif d'économie linguistique.

Le sigle PMA est rattaché à des «critères » (extraits 5, 6, 7, 8, 9) qu'un pays doit remplir pour «être reconnu comme PMA» $(5,8)$, c'est-à-dire obtenir le "statut de PMA» (7) et apparaître dans la «liste» $(6,7)$ réactualisée tous les trois ans : 
(5) Afin d'être reconnu comme PMA, un pays doit remplir les trois critères suivants $[\ldots] .{ }^{11}$

(6) À la lumière des critères utilisés pour établir la liste des PMA (voir annexe 1), il ressort que ces pays sont définis comme des pays à faible revenu, souffrant de grandes faiblesses tant en matière de capital humain que de structure économique. De nombreuses mesures internationales d'appui ont été proposées aux PMA. ${ }^{12}$

(7) L'argument avancé est qu'en établissant un lien plus étroit entre les difficultés structurelles que connaissent les différents pays les moins avancés, par rapport aux critères pris en compte pour le statut de PMA et le traitement spécial accordé à ces pays en vertu de leur statut, on augmenterait l'efficacité de ce dernier. Il est également souligné que, s'il est souhaitable que les pays parviennent à être radiés de la liste des PMA, il importe de prendre à leur égard [...] des mesures internationales d'appui qui auront un effet durable sur la structure de l'économie. ${ }^{13}$

(8) Pays les moins avancés (PMA) : Groupe des pays en développement les plus pauvres. 50 pays, reconnus comme étant des $P M A$, selon des critères établis par l'Assemblée générale des Nations Unies, présentent des économies particulièrement faibles, d'importantes carences en ressources institutionnelles et humaines, et souvent des désavantages géographiques. ${ }^{14}$

Les critères définitoires de la catégorie confèrent aux entités qui la composent un statut de «groupe» (8), présenté comme objectif par l'énoncé d'évaluations quantitatives ou de mesures chiffrées. L'appartenance à la catégorie des PMA est évaluée à partir de trois critères 15 définis sur la base d'indicateurs calculés en dollars, en pourcentages ou en moyennes :

(9) Critères : Faible revenu - 2002 : PIB par habitant [...] Faible capital humain 2002 : indice du capital humain [...] Structure économique insuffisante - 2002 : indice de vulnérabilité économique. ${ }^{16}$

Le codage du sigle rattaché à l'évaluation chiffrée contribue à la production d'une énonciation objective à l'instar des symboles ou abréviations en usage dans le domaine scientifique (Percebois, 2009, p. 20).

En (10) et (11), la forme siglée est actualisée en première occurrence suivie de la forme longue dans «l'espace du décroché» que constituent les parenthèses (usage inversé de celui analysé supra - voir «Le sigle en usage “décroché” ») :

11. ONU [http://www.un.org/fr/], Questions thématiques > Pays les moins avancés.

12. CNUCED, 2002, p. 3 (cité note 7, supra).

13. Ibid., p. 1.

14. $\mathrm{ACICl}$ (Agence de coopération et d'information pour le développement international), Glossaire des termes les plus usités dans le commerce international et en particulier au sein de l'OMC, 2003, Genève [http://www.acici.org/glossaire.htm].

15. Les modalités d'évaluation des trois critères ont évolué au fil des années, hormis celui du PIB (produit intérieur brut) par habitant pour le critère de « faible revenu».

16. CNUCED, 2002, p.14. Tableau en annexe du document : «Évolution de la méthode appliquée pour identifier les PMA». 
(10) Selon la note du président, tous les PMA (pays les moins avancés) seraient automatiquement éligibles en tant qu'importateurs, alors que d'autres membres [de l'OMC] seraient éligibles à la suite d'une notification unique à l'OMC. ${ }^{17}$

(11) L'ancienne DIT [Direction internationale du travail] n'a cependant pas disparu : les PMA (Pays les Moins Avancés) exportent toujours des produits de base. ${ }^{18}$

Cet usage est réglementaire dans la langue française : «Généralement le sigle ou acronyme précède régulièrement son développement. " (Mortureux, 1994, p.18) Lorsque l'on réfère au processus de création du sigle, c'est l'ordre inverse qui prévaut comme en (3) et (5). En plaçant le sigle en première occurrence (10 et 11), les locuteurs font état d'une prévalence supposée partagée de la forme par la communauté des lecteurs de ces bulletins électroniques. La mention de la forme développée n'est là que comme mémo, pour rappel à la mémoire discursive. Les parenthèses jouent ici le rôle d'un marqueur métalinguistique de reformulation (Mortureux, 1993, p.124) qui pourrait se gloser à l'aide du connecteur ou : PMA ou pays les moins avancés. Dans ces emplois, en sus de sa fonction véhiculaire, le sigle remplit donc également la fonction que Jacqueline Percebois nomme la "fonction dénominative lapidaire grégaire» (Percebois, 2001, p. 632) qui rattache la désignation à la «langue spécialisée »19 des institutions, bien connue du lecteur de rapports institutionnels qu'on sait familier avec la terminologie en usage.

À l'évidence, l'usage préférentiel du sigle ne relève pas d'une nécessité d'économie graphique mais bien plutôt de la sélection d'une potentialité signifiante du sigle qui convient mieux au plan de la réalité désignée et au contexte du propos tenu. Ce ne sont pas les entités pays en tant que telles qui sont désignées par le sigle, mais le groupe des membres d'une catégorie établie selon les critères onusiens et, de ce fait, bénéficiant d'aides spécifiques.

Dans les extraits (2) à (11), le sigle PMA est rattaché à des pratiques de classement, d'évaluation, d'attribution de droits propres à l'institution. Il désigne une catégorie constituée d'un ensemble d'entités bénéficiaires d'une aide au développement spécifique, évaluée à l'aune de leur haut degré de difficulté. En (6), (7) et (8), en revanche, la forme développée pays les moins avancés désigne bien les pays²0, c’est-à-dire des territoires peuplés d’humains qui, dans l'agencement énonciatif, sont placés en actants de type «expérient »21

17. ICTSD (International Centre for Trade and Sustainable Development), «ADPIC. Vers un compromis sur les médicaments après la mini-ministérielle de Sydney », Passerelles, vol. I, nº 9, novembre 2002, p.10 [http://ictsd.org/i/news/passerellessynthese/8344/].

18. Skyminds, "La théorie de l'échange» [http://www.skyminds.net/economie-et-sociologie/ ouverture-internationale-et-mondialisation/la-theorie-de-lechange/].

19. «Langue naturelle considérée en tant que vecteur de connaissances spécialisées » (Lerat, 1995, p. 20).

20. Pays : «Division territoriale habitée par une collectivité, et constituant une entité géographique et humaine» (TLFi).

21. Expérient: «le siège d'une perception ou d'un sentiment» (Lazard, 1994, p. 64). 
de procès de 〈souffrir de grandes faiblesses〉 en (6), 〈connaître des difficultés〉 en (7) et 〈présenter d'importantes carences en ressources institutionnelles et humaines > en (8). Seules les collectivités territoriales et humaines que sont les pays connaissent des difficultés et souffrent; le sigle n'atteste que de la capacité de ces pays à recevoir « un traitement spécial» que n’ont pas les autres pays en voie de développement. Le lexème pays est d'ailleurs repris anaphoriquement de l'emploi premier de la forme développée lorsqu'il s'agit d'un acte de radiation (9) que des gouvernements sont seuls à même d'effectuer. En revanche, c'est le sigle qui est actualisé comme mention du statut catégoriel institutionnel. Par métonymie, le sigle devient le support dénominatif des pays en tant que bénéficiaires, comme en (6), (7), (10), (11).

\section{L'usage du sigle opaque comme évitement dans le discours politique}

Comme observé en (4), la forme développée «pays les moins avancés» est susceptible de subir des opérations de défigement qui déforment le syntagme objet de la siglaison. Le sigle en revanche est une forme figée qui n'offre aucune prise à la déformation.

Dans les discours politiques, le syntagme est déformé par une modalité autonymique qui met à distance la caractérisation évaluative infériorisante «les moins avancés » comme dans les exemples (12), (13), (15):

(12) Je souhaite également attirer l'attention de la délégation sur la proposition E 1575, dont l'objet est d'accorder un accès complètement libre au marché communautaire pour les productions des pays dits «les moins avancés». Voici en quelques mots de quoi il s'agit. L'Organisation mondiale du commerce (OMC) a arrêté en 1996 un plan d'action destiné à améliorer l'accès des pays en développement les moins avancés (PMA) aux marchés des pays industrialisés. Ce plan recommande notamment aux pays industrialisés d'octroyer aux produits originaires des $P M A$ un accès en franchise de droits. ${ }^{22}$

(13) La priorité doit être donnée aux besoins immédiats des Petits États insulaires en développement, en particulier ceux du groupe des Pays dits les moins avancés (PMA). Le fonds PMA est un exemple classique d'un fonds pas programmé dans le temps, qui entrave la réussite d'actions appropriées d'adaptation au changement climatique pour les $P M A$. La reconstitution immédiate du fonds $P M A$ est critique pour la mise en œuvre d'actions urgentes d'adaptation pour les 49 PMA. ${ }^{23}$

22. "Communication de M. Jean Bizet sur le texte relatif à l'accès des pays les moins avancés au marché communautaire », Sénat, 2000 [http://213.30.147.231/europe/ro8112000.html\#toc6].

23. "Un résultat ambitieux, qui apportera l'espoir à tous les citoyens du monde» : discours prononcé parEdward Nikape Natapei au Sommet mondial sur le climat, 17 décembre 2009 [http:// www.temoignages.re/un-resultat-ambitieux-qui,41096.html]. 
L'actualisation du participe passé du verbe de parole dire vient déformer la dénomination syntagmatique. Elle désolidarise le lexème pays de l'épithète à valeur scalaire : les moins avancés, qui est représenté comme le dit d'un autre. En (11) et (14), elle est surmodalisée par le «tracé de frontières» (Authier-Revuz, 1995, p. 268) que réalisent les guillemets qui isolent graphiquement le discours autre.

La modalité autonymique permet d'actualiser une forme à la fois en usage et en mention : " [C'est] une forme de dédoublement opacifiant du dire [qui] présente structurellement, le cumul d'une référence à la chose et d'une référence au mot par lequel est nommée la chose.» (Authier-Revuz, 2003, p. 88-89) Par la mise en mention, le locuteur commente son dire par un report de sa responsabilité sur un autre énonciateur qui serait premier. Il marque ainsi sa distance à l'égard d'une dénomination dont il fait néanmoins usage. La modalité autonymique est ici l'indicateur de la gêne - voire du malaise - du locuteur confronté à une dénomination qui, malgré l'euphémisation, traduit l'infériorité de la catégorie de pays ainsi nommée et, de manière plus ou moins directe, celle de ses habitants :

(14) Les Pays les moins avancés... la formule n'est pas très jolie. Elle désigne de manière à peine détournée tout simplement les pays les plus pauvres de la planète. ${ }^{24}$

Comme observé en (7), l'emploi de la forme développée pays les moins avancés actualise le lexème pays qui réfère à des groupes d'humains qui se voient ainsi caractérisés de manière évaluative sur l'échelle du progrès. La qualification d'une avancée est dépréciée par le marqueur de scalarité moins ${ }^{25}$. La dénomination euphémisante "pays les moins avancés» active le programme de sen ${ }^{26}$ du retard de la collectivité sociale des humains qui constituent lesdits pays. Bien qu'euphémisé par égard pour les acteurs sociaux concernés ${ }^{27}$, ce programme de sens reste néanmoins attaché à l'interdiscours civilisateur propre à l'idéologie coloniale.

L'énonciation de ce programme de sens trouve son écho dans les occurrences cotextuelles (pauvres, faible, vulnérable, bas, handicapés) qu' "attire» la forme syntagmatique et qui contribuent à la production de la représentation collective :

(15) «Les pays les moins avancés» (PMA) incluent les 49 pays les plus pauvres de la planète dans quatre continents dont l'Océanie. Selon la CNUCED, ces pays dits «moins avancés» répondent à trois critères : un revenu national bas, un capital

24. Françoise Robert, «Donner une chance aux Pays les moins avancés », En marche [http://www. enmarche.be/International/Politique\%2ointernational/Pays_les_moins_avances.htm].

25. Sur la construction linguistique et discursive de ce marqueur de scalarité, voir notamment Dufour, 2010, p. 208-222.

26. Le programme de sens est le « correspondant dynamique dans la problématique de la production de sens de l'unité minimale que postule, sous forme de sème ou de trait sémantique, l'analyse structurale» (Siblot, 2001, p. 280).

27. “On euphémise sous la pression d'autrui et à l'intention d'autrui.» (Bonhomme, 2005, p. 240) 
humain faible et une grande vulnérabilité économique. Plus de la moitié de l'Afrique fait partie de cette catégorie. ${ }^{28}$

(16) Les pays les moins avancés (PMA), qui constituent le groupe de pays le plus pauvre et le plus faible au sein de la communauté internationale, sont au nombre de 49. Structurellement handicapés dans leur développement, vulnérables au niveau économique, ils méritent une attention particulière de la part de la communauté internationale. Ces pays bénéficient de mesures d'appui spécifiques, en particulier dans le domaine du financement du développement, mais également dans le cadre du commerce multilatéral. 29

De fait, l'appartenance à la catégorie n'a rien d'enviable, malgré la motivation des bénéfices potentiels qu'apporte le statut PMA à des pays en grande difficulté :

(17) En quelques années, la Guinée équatoriale est passée du statut de pays moins avancé (PMA) à celui d'émirat pétrolier aux richesses inouïes. ${ }^{30}$

(18) Certains pays, pour préserver leur image, refusent d'intégrer le groupe. ${ }^{1}$

(19) Ce n'est pas une fierté que d'être inscrit sur cette liste des Pays les moins avancés! Il y a des avantages mais je souhaite que ceux-ci ne soient pas éternels et qu'ils puissent permettre à mon pays de sortir de sa situation de pauvreté. Je serai alors très heureuse et très fière. 32

L'usage de la modalité autonymique est un acte de délocalisation de la responsabilité du dire sur un énonciateur qui pourrait être la communauté discursive $d u$ « on dit» restreinte à des milieux «bien informés». Cette modalité autonymique est très fréquente dans les discours des hommes politiques qui usent de la dénomination sans pour autant en assumer totalement les effets de sens négativants (Dufour, 2009, p. 67-69). L'écho de l'infériorité est difficile à assumer, que l'énonciateur s'identifie comme représentant de la catégorie ou qu'il s'adresse directement ou indirectement à ses représentants comme dans les exemples (11), (12), (14). Alors que la modalité autonymique permet au locuteur de se dédire partiellement de son acte énonciatif, le recours au sigle comme preuve d'une certification validée par un acteur institutionnel permet d'échapper à l'énonciation d'un discours susceptible de «blesser la face».

En (12), Jean Bizet, sénateur de la Manche, modalise la première occurrence de la dénomination pour ensuite actualiser la forme officielle complète

28. Andrea Gibbons, «En marge. Les pauvres dans les PMA à l'ère du commerce mondial», Chronique ONU, vol.XLI, nº3, 2004 [http://www.un.org/french/pubs/chronique/2004/ numero3/0304p35.html].

29. ONU, 2009 [http://www.un.org/fr/globalissues/ldc/index.shtml].

30. Pascal Airault, «Du bon usage du brut», Jeune Afrique, 21 février 2005.

31. Valérie Michaux, «Pays moins avancés. En sortir ou y rentrer?», Syfia Info, $1^{\text {er }}$ mai 2001 [http:// www.syfia.info/index.php5?view=articles\&action=voir\&idArticle=1186].

32. Rabiatou Serah Diallo, secrétaire générale de la Confédération nationale des travailleurs de Guinée, citée par Françoise Robert, En marche, site mentionné supra. 
d'origine : "pays en développement les moins avancés (PMA)», activant le lien avec la source de la catégorisation (ONU, 1970). Cet ajout du sigle en mention rattache implicitement le syntagme à l'énonciateur premier non nommé et décharge ainsi l'énonciateur second (Bizet) de la responsabilité de son acte de nomination. Ce qui lui permet ensuite en troisième occurrence d'actualiser la forme opaque PMA en usage.

L'énonciateur de (13) est Premier ministre de la République de Vanuatu, archipel de la zone Pacifique qui compte parmi les quarante-neuf PMA. Son discours traduit la difficulté à dire que peut ressentir un représentant de cette catégorie de pays à l'énonciation de la dénomination infériorisante. Le sigle PMA en incidente parenthétique constitue une forme de commentaire que l'énonciateur ajoute à la dénomination longue qu'il vient de mettre à distance par la modalité autonymique. L'actualisation du sigle est un acte de renvoi à l'expérience de certification institutionnelle. Sa reprise en épithète «fonds PMA» identifie le sigle à des programmes d'aide internationale. L'introduction du sigle en « décroché» relayé par la référence à un «fonds PMA » autorise ensuite l'usage systématique du sigle pour désigner les pays qui bénéficient de ce fonds. La référenciation à la réalité bien spécifique du fonds lève l'ambiguïté attachée à la forme longue et au sens parasitant produit par la caractérisation «moins avancés».

Le recours au sigle par son pouvoir opacifiant ne donne pas à entendre les programmes de sens à polarité négative. Le sigle, forme neutralisée par son absence de résonance signifiante, permet d'éviter la remontée de la forme source. Il constitue un des moyens d'échapper à la production du sens de la dénomination dévalorisante. En outre, par l'usage du sigle, le locuteur affiche une signature reconnue et partagée par la communauté discursive qui lui permet de se dégager implicitement de la responsabilité pleine et entière de l'énonciation.

La siglaison PMA est rarement justifiée par une reprise moins coûteuse en espace textuel. Les usages analysés ici montrent que les actualisations discursives du sigle et de la forme syntagmatique activent des liens expérientiels différents qui participent de la production de sens différents.

La forme développée pays les moins avancés est rattachée à l'expérience des entités pays en tant que collectivités souffrant d'insuffisances économiques, alors que la forme siglée est plus directement rattachée au statut institutionnel et aux fonds d'aide spécifiques. Le sigle en incidente parenthétique, souvent non repris dans le fil du discours, est une forme de signature qui authentifie son appartenance à l'univers du discours institutionnel. Elle autorise la délocalisation de la fonction d'énonciateur. Son usage privilégié en première occurrence fait état de la pratique de la langue spécialisée en usage dans la communauté discursive.

Quand les discours sont le fait de sujets locuteurs qui parlent en leur nom propre, en d'autres termes des sujets auteurs, l'énonciation du signifiant 
opaque permet de ne pas produire l'écho de mémoire des programmes de sens attachés à la caractérisation dévalorisante les moins avancés en ne transitant pas par la collectivité humaine que constitue le pays. Le processus d'«affectation à une séquence de la forme d'un mot d'une valeur sémantique explicitée par son développement» (Mortureux, 1994, p. 17) propre à la siglaison ne garantit pas une identité de signifiance aux deux formes (le sigle et son syntagme source). Même si le signifié originel peut être restitué par le biais de l'énoncé de la forme syntagmatique qui en est la source, la provision du nouveau signifiant qu'est le sigle offre une potentialité de signifier différemment de la forme source en opérant une catégorisation rattachée à une énonciation datée. Cette potentialité de la siglaison s'explique par le fait que la siglaison réalise un acte de nomination à part entière : "le sigle prend alors valeur de signe» (Calvet, 1980, p. 78). Le néologisme du signifiant produit par le processus de siglaison met à disposition une nouvelle forme dont l'opacité se prête à un codage terminologique qui capitalise les propriétés catégorielles nécessaires et suffisantes telles que définies par l'institution, et seulement elles.

L'acte de catégorisation réalisé par la siglaison PMA procure une alternative d'usage à la forme développée pays les moins avancés. Alors que la forme développée est potentiellement apte à activer des expériences enregistrées en mémoire discursive, la forme opaque du sigle permet aux locuteurs de véhiculer un procès de catégorisation notoirement validé antérieurement par un grand énonciateur institutionnel. Pour cette raison, le sigle, comme alternative signifiante, constitue une modalité de distribution de l'acte d'énonciation qui permet au locuteur de se dissocier énonciativement de son acte de nomination.

\section{Références}

AUTHIER-REVUz Jacqueline, 1995, Ces mots qui ne vont pas de soi. Boucles réflexives et non-coïncidences du dire, Paris, Larousse.

- 2003, «Le fait autonymique : langage, langue, discours. Quelques repères», Parler des mots. Le fait autonymique en discours, J. Authier-Revuz, M. Doury, S. ReboulTouré éd., Paris, Presses de la Sorbonne nouvelle, p. 67-96.

Bon homme Marc, 2005, Pragmatique des figures du discours, Paris, Champion.

CALVEt Louis-Jean, 1980, Les sigles, Paris, PUF.

COSERIU Eugenio, 2001, L'homme et son langage, Louvain, Paris, Peeters.

Dufour Françoise, 2009, "De l'effet de l'interdiscours colonial dans la production du sens de la nomination "pays dits les moins avancés" ", Le sens c'est de la dynamique!, V. Doubli et al. éd., Montpellier, Presses universitaires de la Méditerranée, p. 61-73.

- 2010, De l'idéologie coloniale à celle du développement. Une analyse du discours, Paris, L'Harmattan.

Goffman Erving, 1981, Façons de parler, Paris, Minuit. 
LACHARRIĖRE Guy (de), 1971, "Organisations internationales universelles. Identification et statut des pays "moins développés" ", Annuaire français de droit international, vol.XVII, nº 1, p. 461-482.

LAUNAY Michel, 1986, «Effet de sens : produit de quoi ? ", Langages, n82, p.13-39.

LAZARD Gilbert, 1994, L'actance, Paris, PUF.

LE MONDE, 2002, Le style du Monde, Paris, Le Monde.

LERAT Pierre, 1995, Les langues spécialisées, Paris, PUF.

Mortureux Marie-Françoise, 1993, "Paradigmes désignationnels», Semen, n8, p. 123-141.

- 1994, "Siglaison-acronymie et néologie lexicale», Linx, n³ 30, p. 11-30.

Percebois Jacqueline, 2001, "Fonctions et vie des sigles et acronymes en contextes de langues anglaise et française de spécialité», Meta. Journal des traducteurs, vol.XLVI, $n^{\circ} 4$, p. $627-645$.

— 2009, «Les procédés d'économie linguistique. Quels partenaires pour la communication?», Langue et culture. Mariage de raison?, R. Greenstein éd., Paris, Publications de la Sorbonne, p. 17-43.

- à paraître, «Étude diachronique des termes clés de l'économie du développement en anglais et en français. Entre réalisme et euphémisation », Empreintes de l'euphémisme, Tours et détours, D. Jamet, M. Jobert éd., Paris, L'Harmattan, p. 183-208.

PÉTILLON-BOuCheron Sabine, 2003, Les détours de la langue. Étude sur la parenthèse et le tiret double, Louvain, Paris, Peeters.

SiBlot Paul, 2001, Termes et concepts pour l'analyse du discours, C. Détrie, P. Siblot, B. Vérine éd., Paris, Champion (voir en particulier : «Nomination», p. 205-207, et «Programme de sens», p. 280). 